\title{
$\angle S$ Research Square \\ Finite Element Analysis of the Indirect Reduction of Posterior Pedicle Screw Fixation for a Thoracolumbar Burst Fracture
}

\section{Yuanzheng Song}

Tengzhou Central People's Hospital Affiliated to Jining Medical University

\section{Wei Li}

Tengzhou Central People's Hospital Affiliated to Jining Medical University

Fahao Zhu ( $\square$ tzzxrmyy2013@163.com )

Tengzhou central People's Hospital Affiliated to Jining Medical University https://orcid.org/0000-00019579-3477

\section{Research article}

Keywords: Burst fracture, internal fixators, finite element analysis, indirect reduction

Posted Date: December 18th, 2020

DOI: https://doi.org/10.21203/rs.3.rs-129868/v1

License: (a) (1) This work is licensed under a Creative Commons Attribution 4.0 International License. Read Full License 


\section{Abstract}

Background: Because burst fractures often involve damage to the column and posterior structures of the spine, the fracture block may invade the spinal canal and compress the spinal cord or the cauda equina, causing corresponding neurological dysfunction. When a thoracolumbar burst fracture is accompanied by the presence of bone in the spinal canal, whether posterior surgery requires spinal canal incision decompression remains controversial.

Methods: Computed tomography (CT) images of the thoracolumbar spine of a 31-year-old male with an L1 burst fracture and Mimics 10.0 were used to establish a three-dimensional fracture model for simulating the indirect reduction process. The model was imported into Ansys 10.0, and a $1-10 \mathrm{~mm}$ displacement was loaded $10^{\circ}$ behind the Z-axis on the upper endplate of the L1 vertebral body to simulate position reduction and open reduction. The displacement and stress changes in the intervertebral disc, fractured vertebral body and posterior longitudinal ligament were observed during reduction.

Results: Under a displacement loaded $10^{\circ}$ behind the Z-axis, the maximum stress in the vertebral body was concentrated on the upper disc of the injured vertebrae. The maximum displacement corresponded to the anterior edge of the vertebral body of the injured vertebrae, and the vertebral body height and the anterior lobes were essentially restored. When the displacement load was applied in the positive Z-axis direction, the maximum displacement corresponded to the posterior longitudinal ligament behind the injured vertebrae. Under a $6 \mathrm{~mm}$ load, the posterior longitudinal ligament displacement was $11.3 \mathrm{~mm}$. Under an $8 \mathrm{~mm}$ load, this displacement significantly increased to $15.0 \mathrm{~mm}$, and the vertebral stress was not concentrated on the intervertebral disc.

Conclusions: The reduction of thoracolumbar burst fractures by positioning and distraction allowed the injured vertebrae to be restored to the normal height and kyphotic angle. The reduction of the posterior longitudinal ligament can move the bone block in the spinal canal into the reset space and yield good reset results.

\section{Background}

Thoracolumbar fractures account for approximately $90 \%$ of spinal injuries [1], and $10-20 \%$ of thoracolumbar fractures are burst fractures [2,3]. Because burst fractures often involve damage to the column and posterior structures of the spine, the fracture block may invade the spinal canal and compress the spinal cord or the cauda equina, causing corresponding neurological dysfunction [4]. Because of differences in the degree of fracture and the degree of completeness of neurological function, the optimal treatment for thoracolumbar burst fractures remains controversial $[5,6]$. In recent years, with the development of posterior pedicle fixation devices, posterior pedicle screw fixation has been widely used clinically and has yielded good clinical results. 
Short-segment posterior pedicle treatment for thoracolumbar burst fractures has the advantages of being a relatively simple surgical operation, while causing less trauma and fewer postoperative complications [7-10]. However, when a thoracolumbar burst fracture is accompanied by the presence of bone in the spinal canal, whether posterior surgery requires spinal canal incision decompression is still controversial. In the treatment of patients with impaired neurological function, direct decompression can directly reduce the bone mass in the spinal canal, maximally increasing the volume of the spinal canal; however, the operation time and surgical risks also increase, and this approach causes more postoperative complications than indirect reduction. Therefore, indirect reduction can be used in patients with complete neurological function or mild neurological dysfunction, wherein the bone occupies less than $50 \%$ of the spinal canal and the posterior longitudinal ligament structure is intact.

In intraoperative fluoroscopy, if there is a linear shadow on the posterior edge of the spinal canal in the standard lateral position, spinal canal decompression can be omitted. Yang et al. [11] performed indirect reduction on 64 patients with thoracolumbar burst fractures. The neurological function of all patients was significantly improved, except for 3 patients who were classified as American Spinal Injury Association (ASIA) Grade A before the surgery.

The indirect reduction method for posterior pedicle fixation does not destroy the normal structure of the spinal canal or the posterior structure of the vertebral body. Furthermore, this method does not need to fuse the relevant segments, and the degree of motion of the spine can be preserved to the greatest extent after surgery. Moreover, indirect reduction has prevented iatrogenic injury of the spinal cord and cauda equina and postoperative scar adhesion and has been widely used in the clinic. However, research on the mechanism and basic theory of this method is relatively lacking. We applied the finite element analysis method to establish a finite element model of an adult $L 1$ fracture and simulated posterior pedicle internal fixation in the finite element model for related mechanical loading. The displacement changes in various parts of the vertebral body and related structures during the resetting process were analysed. Through this analysis, the mechanism of the resetting process was explored, which will play a guiding role in future clinical treatment.

\section{Methods}

\section{Case selection}

The study was approved by the Ethics Committee of Tengzhou Central People's Hospital. A male patient (31 years old) with a typical L1 burst fracture was selected, and informed consent was signed.

\section{Experimental model}

A continuous thin layer scan of the thoracolumbar spine was performed with a 64-row spiral computed tomography (CT) scanner, and the resulting scanned image was saved in DICOM format. The saved DICOM format image was read with Mimics 10.01 software (Materialize, Belgian) to obtain an image of T12-L2. The threshold of the target image was defined by adjusting the difference in the grey values of 
the bone tissue and the surrounding tissue. Image processing was performed with the image patching and erasing function in the software, and the intervertebral disc was filled with the mask editing function to obtain a 3D model of the L1 burst fracture (Fig. 1a-e).

This study used the USS titanium alloy internal fixation system from American Orthodontics. The internal fixation system model was built in ProE 2.0 software according to the specific parameters of the nail rod. The diameter and length of the pedicle screw were $6 \mathrm{~mm}$ and $50 \mathrm{~mm}$, respectively, and the diameter of the longitudinal connecting rod was $6 \mathrm{~mm}$. The posterior longitudinal ligament had a width of $10 \mathrm{~mm}$ and a length equal to the length of the spinal canal. These parameters were saved in STL format and were ready for the next simulation of the fusion fix (Fig. $2 a$ and $b$ ).

In the established 3D model of the L1 burst fracture, the intersection of the vertical extension line of the outer edge of the articular process on T12 and L2 and the midline of the transverse process was the entry point, and the posterior pedicle fixation system model was introduced by rotation and translation to the ideal location (Fig. 3a and b).

The model of the established L1 burst fracture combined with the pedicle internal fixation system was simplified and introduced into the finite element software ANSYS 10.0. The software allowed conventional assignment of cortical bone, cancellous bone, posterior structure, intervertebral disc, ligament, internal fixation material, etc. (Table 1). The contact between the small joints was treated as two friction-free contact surfaces. The entire model had a total of 157,070 elements and 242,962 nodes.

Table 1

Material parameters of various parts of the spine and internal fixation [1-3].

\begin{tabular}{|lll|}
\hline Material & Elastic modulus (MPa) & Poisson's ratio $(\boldsymbol{\mu})$ \\
\hline Vertebral cortical bone & 12000 & 0.3 \\
\hline Vertebral cancellous bone & 345 & 0.3 \\
\hline Vertebral endplate & 12000 & 0.3 \\
\hline Rear structure & 3500 & 0.3 \\
\hline Fibre ring & 450 & 0.3 \\
\hline Nucleus pulposus & 1 & 0.499 \\
\hline Posterior longitudinal ligament & 20 & 0.3 \\
\hline Fibrous tissue & 2 & 0.167 \\
\hline Vertebral bone injury & 1 & 0.3 \\
\hline Internal fixation material & 110000 & 0.3 \\
\hline
\end{tabular}


Load and constraints were applied to the finite element model of the entire L1 burst fracture, and the lower surface of the L2 vertebral body was fixed in three directions (X-axis, Y-axis and Z-axis) to constrain the movement or rotation of the surface. During the simulated postural position reduction and open reduction, the anterior surface of the T12 vertebral body was given a displacement of $10^{\circ}$ behind the Zaxis deviation to restore its physiological lordosis, and the Z-axis displacement was given on the T12 vertebral body to analyse the displacement and stress changes in the corresponding vertebral body, intervertebral disc and posterior longitudinal ligament.

\section{Analysis}

The displacement and stress changes in the fractured vertebral body and adjacent intervertebral disc and posterior longitudinal ligament were observed under different loading directions and displacements.

\section{Results}

\section{Displacement and stress under a back load}

Under the positional reset load (post-extension load), a displacement of 1-10 mm was applied $10^{\circ}$ behind the Z-axis of the lamina on T12. We found that when the displacement load was $6 \mathrm{~mm}$, the anterior lordosis of the spine was basically restored, and the height was almost normal. At this time, the maximum displacement of the vertebral body was $11.3 \mathrm{~mm}$ (Fig. 4a), and the maximum change was observed in the height of the injured vertebra (Fig. 4b). The vertebral body stress, which was $51.8 \mathrm{MPa}$, was concentrated on the upper disc of the injured vertebrae (Fig. 4c). The results show that in the process of reducing the physiological anterior lobes of the fracture, because the stress is concentrated in the upper intervertebral disc, the upper and lower endplates of the vertebral body can be effectively opened so that a certain reset space is generated in the middle of the fractured vertebral body. However, the displacement of the posterior longitudinal ligament is not obvious during this process (Fig. 5). Although there is a certain space in the vertebral body, the restoring force of the posterior longitudinal ligament is lacking, and the bone-restoring force in the spinal canal mainly depends on the restoring force of the intervertebral disc. Appropriate over-reduction can significantly increase the stress in the intervertebral disc from 51.8 MPa under a $6 \mathrm{~mm}$ load to $69.1 \mathrm{MPa}$ under an $8 \mathrm{~mm}$ load (Fig. 6), which also shows that proper over-extension is beneficial to the reduction of the fracture.

Stress and displacement under the load (Z-axis positive direction)

We simulated the process of open-replacement of the instrument in the clinic, loading an upward 1$10 \mathrm{~mm}$ displacement on the Z-axis and finding that the maximum displacement occurred in the posterior longitudinal ligament behind the fractured vertebra (Figs. 7 and 8). When the upward $6 \mathrm{~mm}$ displacement load was applied to the Z-axis, the displacement of the posterior longitudinal ligament was $11.3 \mathrm{~mm}$ (Fig. 7), which allowed the distraction to effectively advance the posterior longitudinal ligament and push the bone block of the spinal canal into the reduction space. When the Z-axis was loaded with an upward $8 \mathrm{~mm}$ displacement load, the displacement of the posterior longitudinal ligament can be significantly 
increased to $15.0 \mathrm{~mm}$ (Fig. 9), which also shows that proper over-opening is beneficial to the reduction of the vertebral body and the bone in the spinal canal.

\section{Discussion}

The finite element method is a mathematical analysis method that can be implemented to solve biomechanical problems. This technique was first applied to the medical field in the 1960s. Belytschko et al. [12] established the three-dimensional finite element model of the spine for the first time in 1972. For 40 years, the finite element method has been widely used in the field of spinal surgery. Finite element analysis of thoracolumbar fracture can provide very meaningful digital orthopaedic data for clinical practice, which is helpful for physicians to make appropriate surgical plans and engineering and technical personnel to improve material properties and optimize designs [13-17].

Based on previous related research [18-20], this study established a finite element model of an L1 burst fracture. Because the finite element model requires the internal structure to be as regular as possible during the meshing process, the model was repaired and slightly modified during the introduction process; however, the basic shape of the structure was basically retained. To simulate the true resetting process in the clinic, we divided the loading into two categories. First, in the process of restoring physiological lordosis, we loaded the displacement from $1 \mathrm{~mm}$ to $10 \mathrm{~mm}$ in the direction of $10^{\circ}$ behind the Z-axis. When the displacement was $6 \mathrm{~mm}$, the injured vertebrae recovered the physiological lordosis, and the leading edge height returned to normal. At this time, the whole vertebral body stress was concentrated on the upper intervertebral disc of the injured vertebra, and the upper intervertebral disc played a major role in the restoration of the injured vertebra under high stress, which can restore the height of the front edge of the injured vertebra, correct the kyphosis angle, and injure during the reduction. A certain reduction space was created in the vertebra, which was beneficial to reset the bone in the spinal canal. Second, in the process of opening and resetting, we applied the displacement load in the positive direction of the Z-axis and found that the place where the displacement changes the most was the posterior longitudinal ligament behind the injured vertebra. Due to the reduction of the posterior longitudinal ligament, a certain thrust was applied to the anterior bone mass, thereby pushing the bone mass in the spinal canal into the previously created reset space. By analysing the results of the two loads, we believe that there were two main forces in the reduction of the bone in the spinal canal. First, in the process of restoring physiological lordosis, the height of the vertebral body, especially the height of the anterior border of the vertebral body, was reset by the pulling force of the intervertebral disc (especially the annulus). At this time, there will be some reset space in the vertebral body. During the distraction process, the bone block in the spinal canal was pushed into the internal space of the vertebral body by the action of the posterior longitudinal ligament, thereby achieving the reset effect. This phenomenon also explains the poor effect of vertebral fracture reduction when the bone was turned over in the spinal canal or accompanied by posterior longitudinal ligament injury. Kose et al. [21] analysed the influencing factors of bone reduction in the spinal canal and found that the height of the vertebral body leading edge and the reduction of the vertebral wedge angle were substantial influencing factors, which is consistent with our research. In addition, the reduction effect of T12 and L1 is better than that of L2, and the reason 
for this discrepancy is related to the anatomy of the posterior longitudinal ligament. Hu et al. [22] found that the posterior longitudinal ligament exhibited the highest toughness in the thoracolumbar region. We believe that the main influencing factors of bone reduction in the spinal canal are the posterior longitudinal ligament and the intervertebral disc (especially the annulus fibrosus). The intervertebral disc plays an important role in reducing the height of the vertebral body and the angle of the wedge. The main function of the posterior longitudinal ligament is to push the bone in the spinal canal into the space created by the reduction of the vertebral body. The indirect reduction of the posterior ligament complex of the spine eliminates the need for extensive incisions to separate muscles, thereby reducing damage to muscle vessels. Furthermore, in this approach, there is less intraoperative bleeding and less trauma, which is conducive to the recovery of lumbar function. Thus, implementing this approach can enable the patient to get out of bed earlier after surgery, thereby satisfying the concept of rapid rehabilitation surgery [23]. In clinical applications, we should pay attention to adjusting the angle of the nail to facilitate the reduction of the lordosis of the vertebral body. In addition, moderate overcorrection can significantly increase the reduction of the disc.

The finite element simulation has certain errors due to the influence factors, such as model simplification and assignment distortion. Due to the limitations in finite element model design experience, the finite element model is not perfect. The limitations in this study are that the effects of the surrounding tissues of the vertebral body (soft tissues, such as muscles) on the reduction process are not considered and that other types of fractures, such as endplate fractures, are not elaborated. In the future, we will continue to improve the process of generating and analysing the model so that the analysis results will be closer to the real environment of the human body.

\section{Conclusions}

This study established a three-dimensional finite element model of an L1 burst fracture and achieved a clinical reduction in subsequent loading. We simulated the operative lordosis and vertebral body distraction by implementing two loads and recorded the deformation and stress in different parts of the vertebral body, especially the intervertebral disc and posterior longitudinal ligament. When the position is reset, we can restore the normal height of the injured vertebrae and correct the kyphosis through stress concentration on the intervertebral disc, creating a certain reset space in the vertebral body. When the instrument is opened, the reduction in the posterior longitudinal ligament can push the bone in the spinal canal into the reduction space, thereby achieving a reset. The implemented loading method closely resembles the clinical application; thus, this study provides certain reference materials for treating such patients in the clinic.

\section{Abbreviations}

CT: Computed tomography; 3D: Three-dimensional; ASIA: American spinal injury association

\section{Declarations}


Ethics approval and consent to participate

The ethics committee of Tengzhou Central People's Hospital approved this study(2020-Ethics review-08). Informed consent was obtained from all individual participants included in the study.

\section{Consent for publication}

Written informed consent was obtained from each patient to authorize the publication of their data.

\section{Availability of data and materials}

The datasets used and analysed during the current study are available from the corresponding author upon reasonable request.

\section{Competing interests}

The authors declare that they have no competing interests.

\section{Funding}

This study received a supporting fund for teachers' research from Jining Medical University (grant no. JYFC2019FKJ187).

\section{Authors' contributions}

Yuanzheng Song and Fahao Zhu designed the study and performed the experiments; Yuanzheng Song, Wei Li and Fahao Zhu performed the experiments, analysed the data, and wrote the manuscript.

\section{Acknowledgements}

We would like to thank Professor Yang HL of the Department of Spine Surgery at the First Affiliated Hospital of Soochow University for his valuable suggestions to improve the analysis and interpretation of the results. We are also grateful to the patient who participated in this study.

\section{References}

1. Kraemer WJ, Schemitsch EH, Lever J, McBroom RJ, McKee MD, Waddell JP. Functional outcome of thoracolumbar burst fractures without neurological deficit. J Orthop Trauma. 1996;10:541-4.

2. Muller U, Berlemann U, Sledge J, Schwarzenbach O. Treatment of thoracolumbar burst fractures without neurologic deficit by indirect reduction and posterior instrumentation: bisegmental stabilization with monosegmental fusion. Eur Spine J. 1999;8:284-9.

3. Esses SI, Botsford DJ, Kostuik JP. Evaluation of surgical treatment for burst fractures. Spine (Phila Pa 1976). 1990;15:667-73. 
4. Denis F. Spinal instability as defined by the three-column spine concept in acute spinal trauma. Clin Orthop Relat Res. 1984;189:65-76.

5. Dai LY, Jiang SD, Wang XY, Jiang LS. A review of the management of thoracolumbar burst fractures. Surg Neurol. 2007;67:221-31; discussion 231.

6. van der Roer N, de Lange ES, Bakker FC, de Vet HC, van Tulder MW. Management of traumatic thoracolumbar fractures: a systematic review of the literature. Eur Spine J. 2005;14:527-34.

7. Li C, Zhou Y, Wang H, Liu J, Xiang L. Treatment of unstable thoracolumbar fractures through short segment pedicle screw fixation techniques using pedicle fixation at the level of the fracture: a finite element analysis. PLoS One. 2014;9:e99156.

8. Chen Z, Wu J, Lin B, Wu S, Zeng W. Posterior short-segment fixation including the fractured vertebra for severe unstable thoracolumbar fractures. Zhongguo Xiu Fu Chong Jian Wai Ke Za Zhi. 2018;32:59-63.

9. Alpantaki K, Bano A, Pasku D, Mavrogenis AF, Papagelopoulos PJ, Sapkas GS, et al. Thoracolumbar burst fractures: a systematic review of management. Orthopedics. 2010;33:422-9.

10. Ayberk G, Ozveren MF, Altundal N, Tosun H, Seckin Z, Kilicarslan K, et al. Three column stabilization through posterior approach alone: transpedicular placement of distractable cage with transpedicular screw fixation. Neurol Med Chir (Tokyo). 2008;48:8-14; discussion 14.

11. Yang HL, Shi JH, Liu J, Ebraheim NA, Gehling D, Pataparla S, et al. Fluoroscopically-guided indirect posterior reduction and fixation of thoracolumbar burst fractures without fusion. Int Orthop. 2009;33:1329-34.

12. Belytschko T, Kulak RF, Schultz AB, Galante JO. Finite element stress analysis of an intervertebral disc. J Biomech. 1974;7:277-85.

13. Fei Q, Li QJ, Li D, Yang Y, Tang H, Li JJ, et al. Biomechanical effect on adjacent vertebra after percutaneous kyphoplasty with cement leakage into disc: a finite element analysis of thoracolumbar osteoporotic vertebral compression fracture. Zhonghua Yi Xue Za Zhi. 2011;91:51-5.

14. Panchal RR, Matheis EA, Gudipally M, Hussain MM, Kim KD, Bucklen BS. Is lateral stabilization enough in thoracolumbar burst fracture reconstruction? A biomechanical investigation. Spine J. 2015;15:2247-53.

15. Zhang H, Guo C, Tang M, Liu S, Li J, Guo Q, et al. Prevalence of scoliosis among primary and middle school students in Mainland China: a systematic review and meta-analysis. Spine (Phila Pa 1976). 2015;40:41-9.

16. Chen HW, Liu GD, Wu LJ. Complications of treating terrible triad injury of the elbow: a systematic review. PLoS One. 2014;9:e97476.

17. Atesok K, Galos D, Jazrawi LM, Egol KA. Preoperative planning in orthopaedic surgery. Current practice and evolving applications. Bull Hosp Jt Dis (2013). 2015;73:257-68.

18. Tang HZ, Xu H, Yao XD, Lin SQ. Single-stage posterior vertebral column resection and internal fixation for old fracture-dislocations of thoracolumbar spine: a case series and systematic review. Eur Spine J. 2016;25:2497-513. 
19. Zibis AH, Mitrousias V, Baxevanidou K, Hantes M, Karachalios T, Arvanitis D. Anatomical variations of the foramen transversarium in cervical vertebrae: findings, review of the literature, and clinical significance during cervical spine surgery. Eur Spine J. 2016;25:4132-9.

20. Jain D, Berven S. Commentary on development and assessment of a digital X-ray software tool to determine vertebral rotation in adolescent idiopathic scoliosis. Spine J. 2017;17:266-8.

21. Kose KC, Inanmaz ME, Isik C, Basar H, Caliskan I, Bal E. Short segment pedicle screw instrumentation with an index level screw and cantilevered hyperlordotic reduction in the treatment of type-A fractures of the thoracolumbar spine. Bone Joint J. 2014;96-B:541-7.

22. Hu Z, Zhou Y, Li N, Xie X. Correlations between posterior longitudinal ligament status and size of bone fragment in thoracolumbar burst fractures. Int J Clin Exp Med. 2015;8:2754-9.

23. Phan K, Rao PJ, Mobbs RJ. Percutaneous versus open pedicle screw fixation for treatment of thoracolumbar fractures: Systematic review and meta-analysis of comparative studies. Clin Neurol Neurosurg. 2015;135:85-92.

\section{Figures}

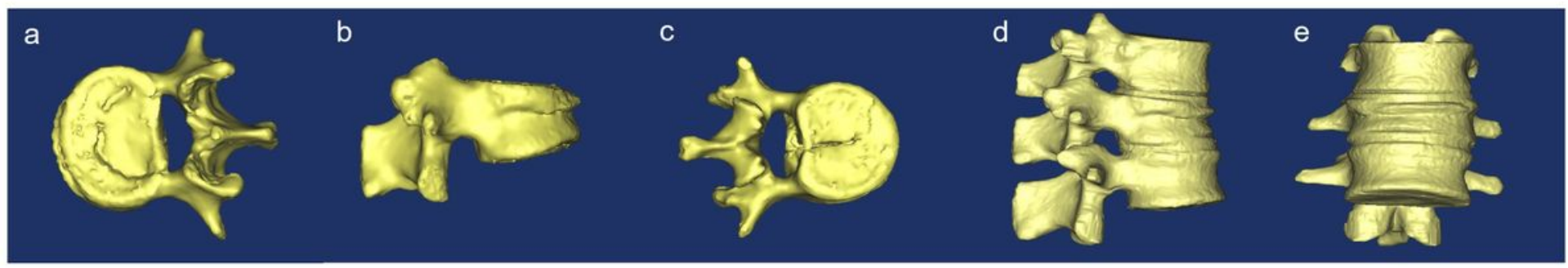

\section{Figure 1}

Establishing a 3D model of an L1 burst fracture: (a) endplate of the fractured vertebral body, (b) side of the fractured vertebral body, (c) lower endplate of the fractured vertebral body, (d) side of the 3D model of the fractured vertebral body and its adjacent vertebral bodies, and (e) front of the 3D model of the fractured vertebral body and its adjacent vertebral bodies. 


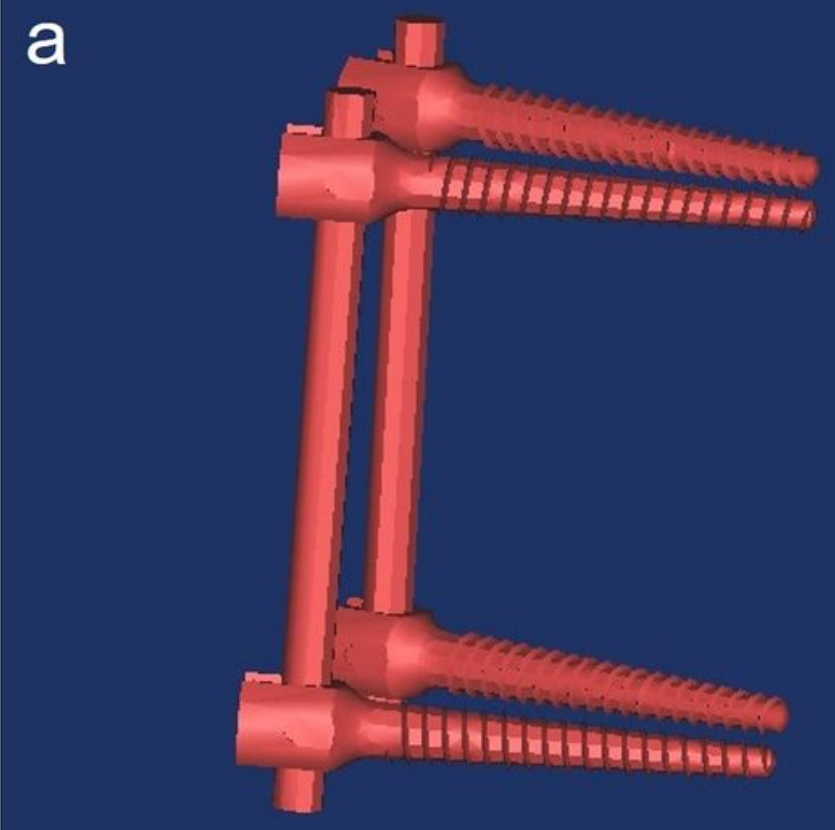

b

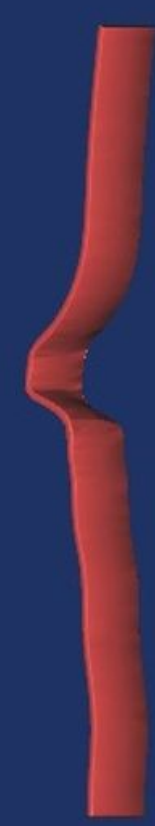

\section{Figure 2}

(a) Establishing a model of the pedicle internal fixation system, including screws and connecting rods. (b) Establishing the T12-L2 posterior longitudinal ligament model.

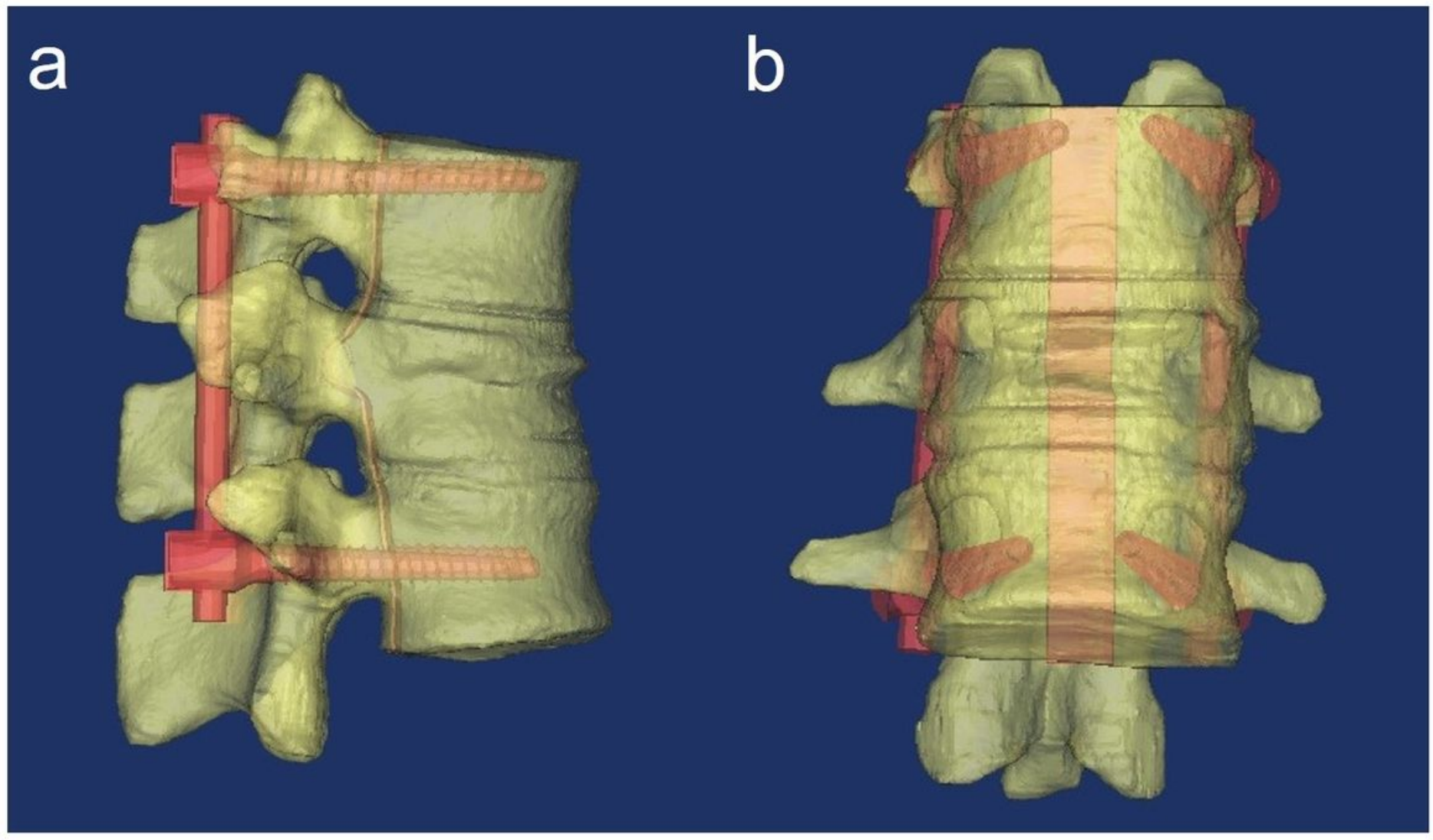

Figure 3 
Establishing a short-segment posterior pedicle internal fixation model: (a) introducing the pedicle internal fixation system into the L1 vertebral burst fracture model and (b) introducing the posterior longitudinal ligament model into the L1 vertebral burst fracture model.

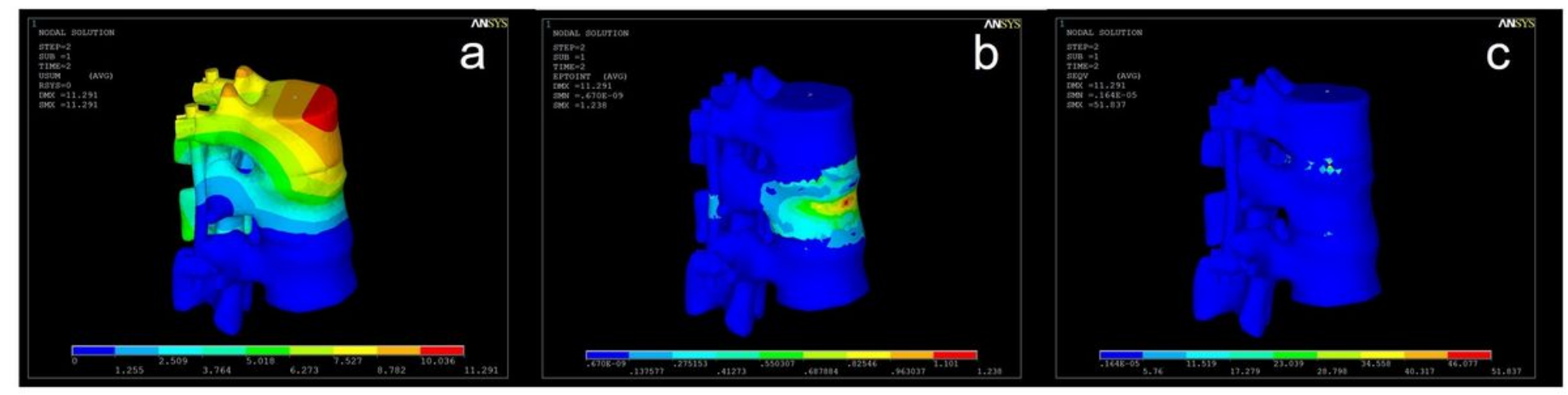

\section{Figure 4}

Deformation of the vertebral body under a $6 \mathrm{~mm}$ extension displacement. (a) When the extensional displacement load is $6 \mathrm{~mm}$, the anterior lordosis of the spine is basically restored, the height is almost normal, and the maximum displacement of the vertebral body is $11.3 \mathrm{~mm}$. (b) The height of the injured vertebra changes the most. (c) The vertebral body stress, which is $51.8 \mathrm{MPa}$, is concentrated on the upper disc of the injured vertebrae. 


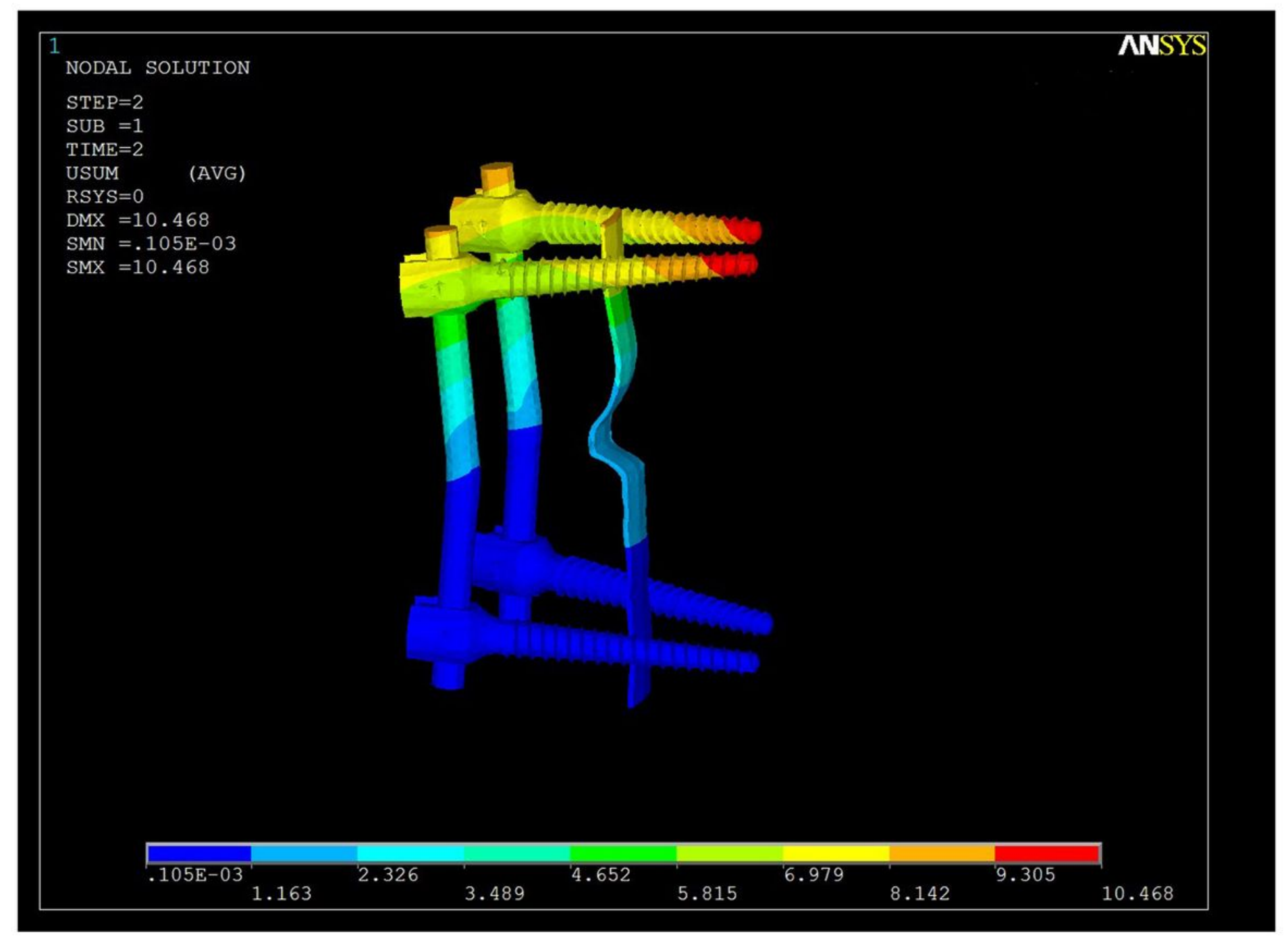

Figure 5

Strain in the pedicle internal fixation device and posterior longitudinal ligament under a $6 \mathrm{~mm}$ extension displacement. The posterior longitudinal ligament displacement is not obvious. 


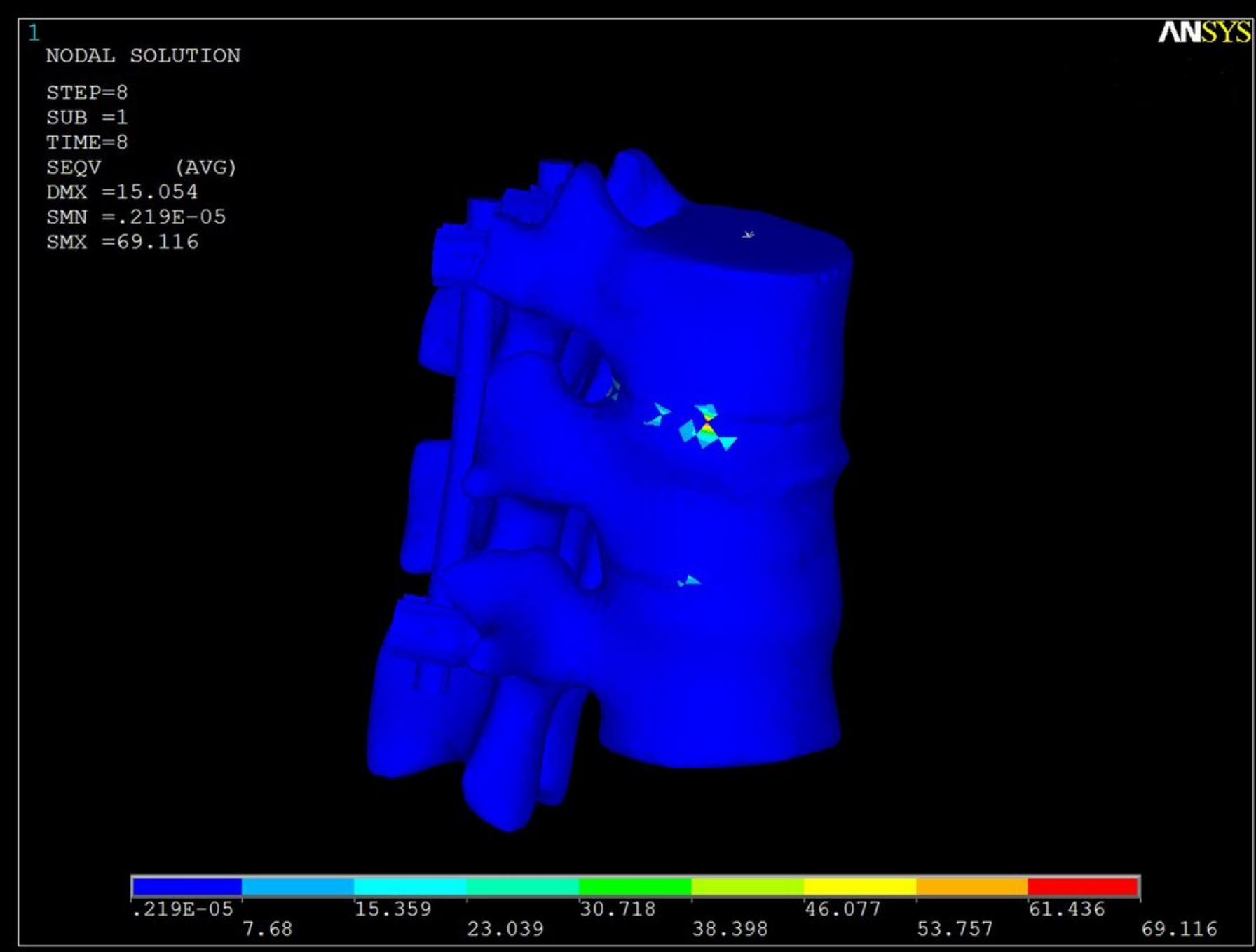

\section{Figure 6}

Vertebrae stress diagram under an $8 \mathrm{~mm}$ extension displacement. Under the $8 \mathrm{~mm}$ extension and displacement load, the vertebral body stress, which is $69.1 \mathrm{MPa}$, is also concentrated on the upper disc of the injured vertebrae. 


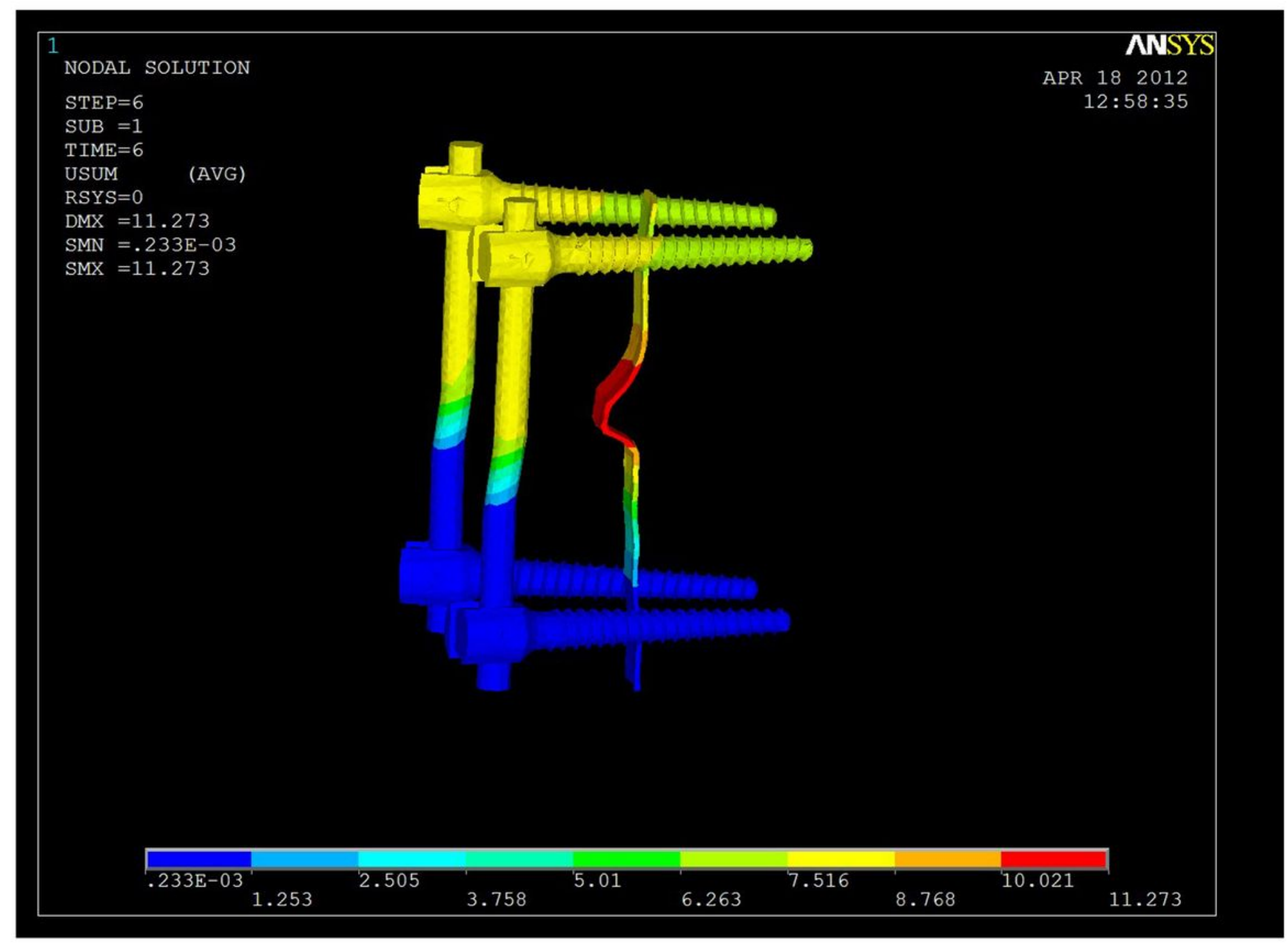

\section{Figure 7}

Deformation diagram of the pedicle and posterior longitudinal ligament model when the distraction displacement is $6 \mathrm{~mm}$ in the positive direction of the Z-axis. The largest change in displacement, which has a value of $11.3 \mathrm{~mm}$, corresponds to the posterior longitudinal ligament. 


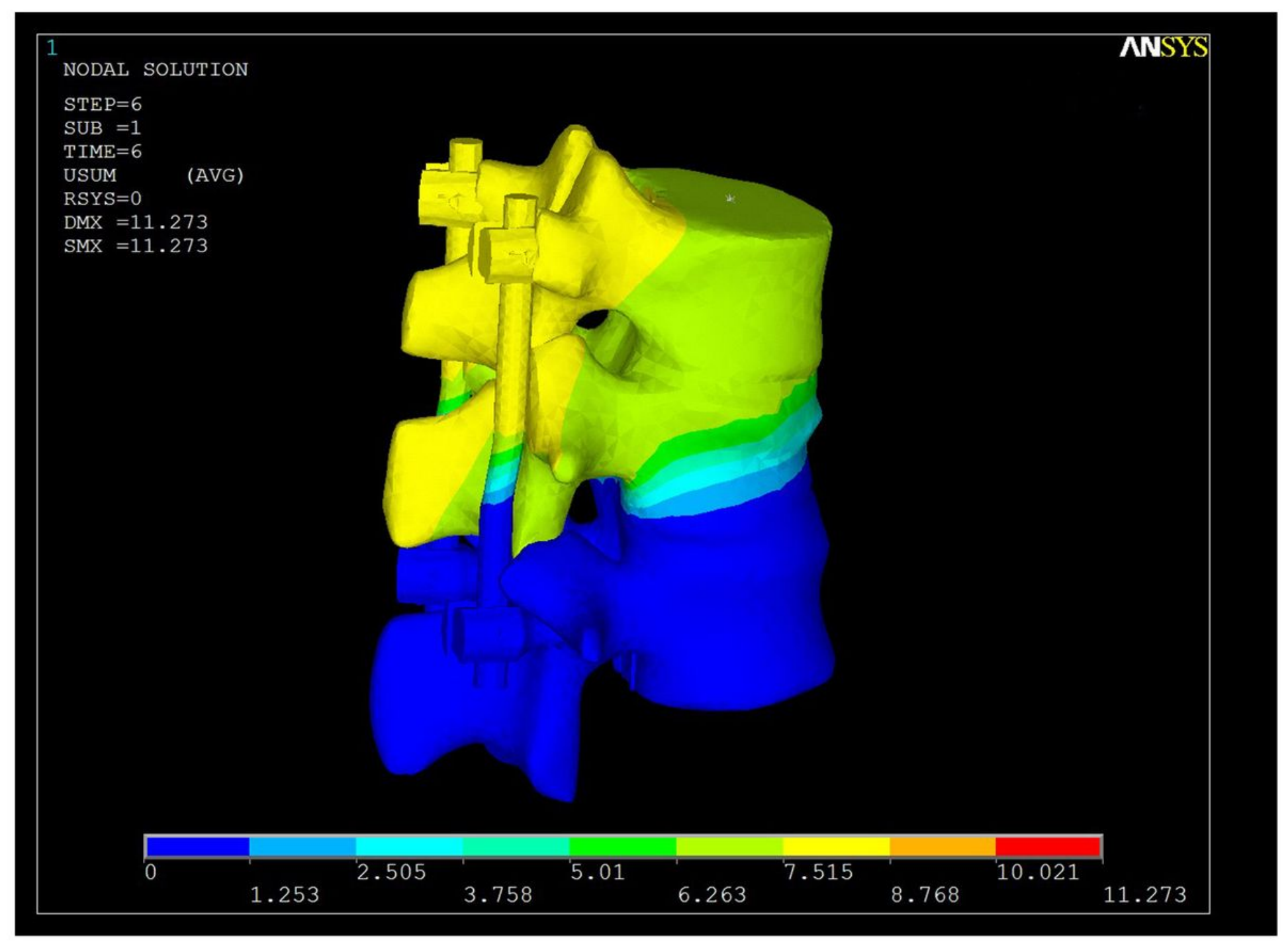

\section{Figure 8}

Deformation diagram of the whole body of the vertebral body when the distraction displacement is $6 \mathrm{~mm}$ in the positive direction of the Z-axis. 


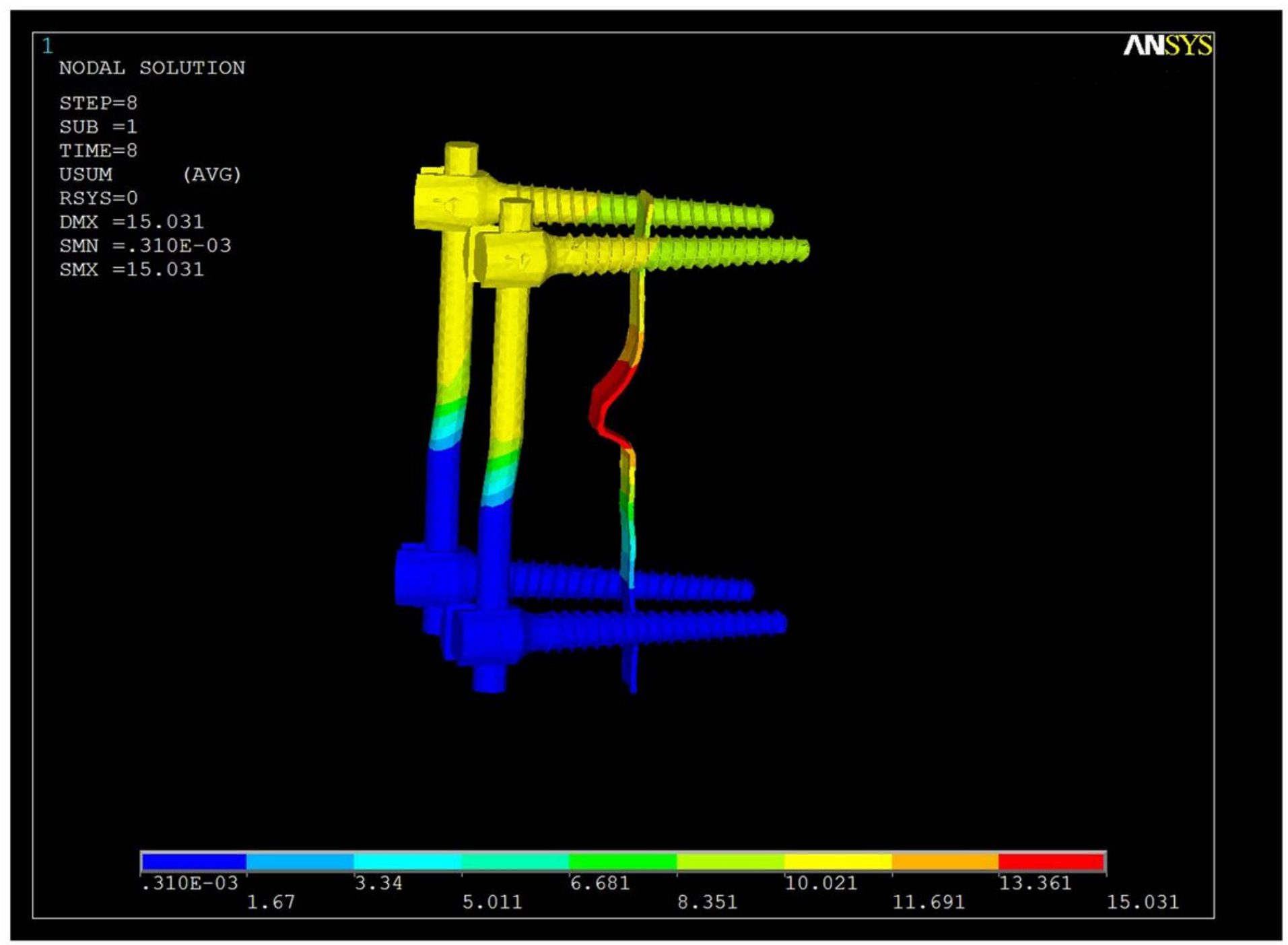

\section{Figure 9}

Deformation diagram of the pedicle and posterior longitudinal ligament model when the distraction displacement is $8 \mathrm{~mm}$ in the positive direction of the Z-axis. The largest change in displacement, which has a value of $15.0 \mathrm{~mm}$, corresponds to the posterior longitudinal ligament. 